\title{
Noncommutative geometry and fluid dynamics
}

\author{
Praloy Das ${ }^{\mathrm{a}}$, Subir Ghosh ${ }^{\mathrm{b}}$ \\ Physics and Applied Mathematics Unit, Indian Statistical Institute, 203 B. T. Road, Kolkata 700108, India
}

Received: 29 August 2016 / Accepted: 7 November 2016 / Published online: 17 November 2016

(C) The Author(s) 2016. This article is published with open access at Springerlink.com

\begin{abstract}
In the present paper we have developed a NonCommutative (NC) generalization of perfect fluid model from first principles, in a Hamiltonian framework. The noncommutativity is introduced at the Lagrangian (particle) coordinate space brackets and the induced NC fluid bracket algebra for the Eulerian (fluid) field variables is derived. Together with a Hamiltonian this NC algebra generates the generalized fluid dynamics that satisfies exact local conservation laws for mass and energy, thereby maintaining mass and energy conservation. However, nontrivial NC correction terms appear in the charge and energy fluxes. Other non-relativistic spacetime symmetries of the NC fluid are also discussed in detail. This constitutes the study of kinematics and dynamics of NC fluid. In the second part we construct an extension of the FriedmannRobertson-Walker (FRW) cosmological model based on the NC fluid dynamics presented here. We outline the way in which NC effects generate cosmological perturbations bringing about anisotropy and inhomogeneity in the model. We also derive a NC extended Friedmann equation.
\end{abstract}

\section{Introduction}

It is very intriguing to learn that there are two parallel languages, fluid mechanics and Non-Commutative (NC) gauge theories, in which several completely unrelated physical systems can be formulated [1-6]. For example the discrete nature of a quantum Hall liquid can be described by NC gauge theories [1-6]. On the other hand, the analogy between diffeomorphism and related transformations in the fluid space and the Seiberg-Witten map [7] in NC gauge theories has led to a series of works by Jackiw et al. [8-11], where these connections are studied in a

\footnotetext{
a e-mail: praloydasdurgapur@gmail.com

b e-mail: subirghosh20@gmail.com
}

deeper perspective. (For a lucid review see [12]). For example magnetohydrodynamics in a very strong magnetic field, where excitations in the lowest Landau level play a dominant role, provides a natural framework of NC gauge theory $[8-11,13]$. The connection between fluids and NC field theories was envisaged much earlier in [14].

The above disconnected ideas have naturally led to attempts to formulate a generalization of canonical perfect fluid theory in NC space(time) where the space(time) is endowed with a noncommutative structure. Incidentally we have put the 'time' in parentheses simply to indicate that conventionally the NC extension is restricted to the spatial sector only without affecting the time. Some relevant work in this context is [15-17].

In this perspective we have provided an explicit model of a generalized perfect fluid living in noncommutative space. We stress that the model is constructed from first principles, completely based on the map between the Lagrangian and Hamiltonian (Euler) formulation of fluid dynamics. The NC effect is induced in the Euler continuum algebra from the NC extension in discrete Lagrangian variable algebra.

There are two parallel ways of constructing an NC extension of a theory. One route (if one is interested in $\mathrm{NC}$ field theory) is to directly apply the Groenwald-Moyal *product [7,18-20] in a conventional model field theory to obtain the NC-extended field theory action. See e.g. earlier reviews [18-20] for generic NC field theory construction and $*$-product formalism in $[15,16]$ for NC fluid model. The other alternative that is especially suited for fluids is to start from the Lagrangian (discrete) fluid model, introduce the NC space effect and subsequently exploit the map that connects the Lagrangian framework to an Euler Hamiltonian (continuum) framework to induce NC effects in the fluid field theory. We have followed the latter approach, the primary reason being that NC generalization can be unambiguously done in the discrete variable setup (see for exam- 
ple [17]). ${ }^{1}$ In the present work we have provided the full NC extended algebra among the Euler degrees of freedom, i.e. the density and velocity field variables, and furthermore we have studied briefly some consequences of it in cosmology. ${ }^{2}$

Let us briefly discuss the recent importance of Hydrodynamics and noncommutative field theory in theoretical high energy physics. Hydrodynamics can be described in very general terms as a universal description of long wavelength physics that deals with low energy effective degrees of freedom of a field theory, classical or quantum. Interestingly it applies equally well at macroscopic and microscopic scales, examples being the liquid drop model in early nuclear physics and quark-gluon plasma produced at RHIC/LHC (in the former) and generic fluid models in cosmology (in the latter). The physical reasoning behind the success of fluid models is the reasonable assumption that at sufficiently high energy densities local equilibrium prevails in an interacting field theory so that local inhomogeneities are smoothed out enough to reduce to continuous fluid variables. Indeed the constitutive relations provide the bridge between the fundamental and fluid degrees of freedom. In the fluid picture one simply deals with the age old continuity equation and the Euler force equation (in the most simple non-relativistic framework for an ideal fluid). Two of the most exciting and topical areas of recent interest are cosmology and the AdS-CFT correspondence [30-32] as applied to the gauge-gravity duality $[33,34]$ and both of them rely heavily on conventional fluid dynamics. Hence it will be very interesting to study the effects of nontrivial changes in fluid equations (at a fundamental level), in these areas. In the present paper we aim to outline how fluid dynamics in non-commutative (NC) space can affect cosmology by introducing cosmological perturbations. We postpone discussions on NC effects induced by fluid dynamics on the gauge-gravity duality to a future publication.

A recently rediscovered effect that has impacted the theoretical physics community in a major way is the idea of introducing a NC spacetime and subsequently a NC generalization of quantum field theory. Seiberg and Witten [7], in their seminal work, resurrected the (not so successful) NC spacetime introduced by Snyder [21-23], when the former demonstrated that in certain low energy limits open string dynamics (with end points on $D$-branes) can be simulated by NC field theories where the NC parameter $\theta_{\mu \nu}$ is to identified with the antisymmetric two-form field $B_{\mu \nu}$.

\footnotetext{
1 In fact the first NC model proposed by Snyder, see also [21-23], was in a discrete framework and later on numerous NC extensions in quantum mechanical models have been proposed [24-29].

2 It should be noted that in the review [12] an NC generalized fluid variable algebra has been outlined starting from the discrete Lagrangian setup but it is not complete since only the NC extension of the densitydensity local algebra has been computed.
}

This idea has led to a huge amount of literature and we refer to some review work in [18-20]. In the present paper we develop a generalized fluid dynamics, compatible with $\mathrm{NC}$ space (since we work in non-relativistic regime), from first principles. One generally tends to avoid introducing $\mathrm{NC}$ between time and spatial degrees of freedom due to possible complications regarding unitarity in the quantum theory.

Before getting down to the actual computation let us pause for a moment to comment on the justification of our scheme of introducing noncommutativity. Conventionally in NC quantum mechanics one simply postulates a generalization of the canonical phase space commutator algebra such that it reduces to the canonical commutators for the vanishing of $\mathrm{NC}$ parameter. From a purely algebraic standpoint the only restriction is that the NC algebra has to satisfy the Jacobi identity (assuming that the canonical commutator algebra does). Subsequent restrictions will come from physical implications of the extra NC terms. Clearly if the NC contribution in the commutator are (non-operatorial) c-number in nature the above condition is satisfied since the NC terms in the double commutators trivially vanish (see for example [24-29]). On the contrary for NC extensions of operatorial form (such as Snyder, $\kappa$-Minkowski, Generalized Uncertainty Principleinduced algebra) the $\mathrm{NC}$ extensions are severely restricted in order to satisfy Jacobi identity (see for example [3542]).

Let us now come back to the classical scenario that is of present interest. Just as classical Poisson algebra is elevated to quantum commutators via the correspondence principle in exactly identical fashion one can think of a classical counterpart of the NC quantum algebra. Once again the Jacobi identity, in the sense of double brackets, plays an essential role, since for a Hamiltonian system the symplectic structure (brackets) has to obey the Jacobi identity. However, there is an added twist in the classical setup where sometimes it is possible to judiciously introduce constraints in a particular model under study such that the NC generalized algebra can be identified with the Dirac bracket algebra (see for example [43-47]), in Dirac's Hamiltonian formulation of a constrained system [48]. The advantages are that (i) one has a Lagrangian for the NC system and (ii) the satisfaction of Jacobi identity is ensured since Dirac brackets satisfy the Jacobi identity.

Let us now outline our formalism following [12]. ${ }^{3}$ The NC fluid model proposed by us rests essentially on the map between the Lagrangian and Eulerian or (Hamiltonian) description of fluid dynamics. The former is based on a microscopic picture where the fluid is treated as a collection (albeit a large number) of point particles obeying canon-

\footnotetext{
3 Recent work using this framework in the context of relativistic fluids is [49-51].
} 
ical Newtonian dynamics. The d.o.f.s consist of the particle coordinate and velocity, $\mathbf{X}_{\mathbf{i}}(t), \mathrm{d} \mathbf{X}_{\mathbf{i}}(t) / \mathrm{d} t$, respectively, where $i$ stands for particle index. In the limit of a continuum of particle d.o.f. these reduce to $\mathbf{X}(\mathbf{x}, t), \mathrm{d} \mathbf{X}(\mathbf{x}, t) / \mathrm{d} t$ with $\mathbf{x}$ replacing the discrete index $i$. On the other hand the Eulerian scheme starts by providing a field theory Hamiltonian $H=$ $\int d^{3} r \mathcal{E}(\rho, \mathbf{v})$ and a set of Poisson brackets between the fluid d.o.f. that are the density and velocity fields $\rho(\mathbf{r}, t), \mathbf{v}(\mathbf{r}, t)$, respectively. The fluid equations of motion are derived from the above as Hamilton's equation of motion. There is a map that connects Lagrangian variables $(\mathbf{X}(\mathbf{x}, t), \mathrm{d} \mathbf{X}(\mathbf{x}, t) / \mathrm{d} t)$ to Eulerian fields $(\rho(\mathbf{r}, t), \mathbf{v}(\mathbf{r}, t))$ and, most importantly, a field theoretic Poisson algebra between the Euler variables is derived from the canonical point mechanics Poisson brackets between Lagrangian variables. (These are discussed later in detail.) This is best suited for our purpose since, as discussed earlier, the NC brackets are given most naturally in a point mechanics framework, that is, in terms of Lagrangian variables. It is worthwhile to recall here that even the canonical point mechanics (Poisson) brackets lead to a quite involved and non-linear set of operatorial algebra between the Euler variables. Hence it is not entirely surprising that the simplest extension of canonical brackets to NC brackets in a Lagrangian setup will lead to an involved and qualitatively distinct NC extended brackets among Euler variables. However, as we will explicitly demonstrate, these NC brackets yield a modified set of continuity equation and Euler force equation with an unchanged conserved mass and energy but $\mathrm{NC}$ corrected mass and momentum fluxes. Clearly these NC modifications will leave their marks on cosmological solutions and in particular the NC corrections will act as specific forms of cosmological perturbations.

The paper is organized as follows: in Sect. 2 we recapitulate the Hamiltonian or Euler form of fluid dynamics along with its spacetime symmetries [12]. Section 3 deals with the NC fluid variable algebra with a discussion of the Jacobi identities concerning the new NC fluid algebra. Section 4 is devoted to a study of the generalized continuity and conservation principles including comments on spacetime symmetries for NC fluid. In Sect. 5 we provide an outline on effects on cosmological principles induced by NC modified fluid system. We conclude in Sect. 6 with a summary of our work and its future prospects.

\section{Canonical fluid and its symmetries in Hamiltonian framework}

Let us start with a quick recapitulation of the formulation and results for a conventional (or canonical) perfect fluid in Euler (or Hamiltonian) formulation [12]. There is a wellknown prescription of starting with a microscopic or discrete picture of dynamics of a system of particles in a Lagrangian framework leading to a continuum field theory of fluid in Eulerian scheme. In fact this basic connection between the two formalisms plays the central role in the consistent generalization of the fluid in noncommutative space.

Newton's law for the particle (Lagrangian) coordinate $X_{i}(t)$ and velocity $v_{i}(t)=\dot{X}_{i}$ is given by

$m \ddot{X}_{i}(t)=m \dot{v}_{i}(t)=F_{i}(X(t))$,

where $m$ is the particle mass and $F_{i}(X(t))$ is the applied force. The Eulerian density field for the single particle is

$\rho(t, \mathbf{r})=m \delta(\mathbf{X}(t)-\mathbf{r})$.

Hence the density and current fields for a collection of particles are given by

$\rho(t, \mathbf{r})=m \sum_{n=1}^{N} \delta\left(\mathbf{X}_{n}(t)-\mathbf{r}\right)$,

$\mathbf{j}(t, \mathbf{r})=\mathbf{v}(t, \mathbf{r}) \rho(t, \mathbf{r})=m \sum_{n=1}^{N} \dot{\mathbf{X}}_{n}(t) \delta\left(\mathbf{X}_{n}(t)-\mathbf{r}\right)$.

For fluid field theory the discrete particle labels are replaced by continuous variables and we end up with

$\rho(\mathbf{r})=\rho_{0} \int \delta(X(x)-r) \mathrm{d} x$,

$v_{i}(\mathbf{r})=\frac{\int \mathrm{d} x \dot{X}_{i}(x) \delta(X(x)-r)}{\int \mathrm{d} x \delta(X(x)-r)}$.

From now on we will omit $t$ from the arguments of the d.o.f. In a Hamiltonian formulation the canonical Poisson bracket structure is given by

$\left[\dot{X}^{i}, X^{j}\right]=(i / m) \delta^{i j}, \quad\left[X^{i}, X^{j}\right]=0$,

$\left[\dot{X}^{i}, \dot{X}^{j}\right]=0$.

For the Lagrangian fluid this is generalized to [12],

$\left\{\dot{X}^{i}(\mathbf{x}), X^{j}\left(\mathbf{x}^{\prime}\right)\right\}=\frac{1}{\rho_{0}} \delta^{i j} \delta\left(\mathbf{x}-\mathbf{x}^{\prime}\right) ;$

$\left\{X^{i}(\mathbf{x}), X^{j}\left(\mathbf{x}^{\prime}\right)\right\}=\left\{\dot{X}^{i}(\mathbf{x}), \dot{X}^{j}\left(\mathbf{x}^{\prime}\right)\right\}=0$.

Obviously the above bracket structure satisfies the Jacobi identity. Using the definitions of $\rho$ and $\mathbf{j}$ in terms of $\mathbf{X}$ and $\dot{\mathbf{X}}$ given above, a straightforward but somewhat nontrivial computation leads to the Poisson algebra between the Euler variables $\rho$ and $\mathbf{j}$ :

$\left\{\rho(\mathbf{r}), \rho\left(\mathbf{r}^{\prime}\right)\right\}=0$,

$\left\{j^{i}(\mathbf{r}), \rho\left(\mathbf{r}^{\prime}\right)\right\}=\rho(\mathbf{r}) \partial_{i} \delta\left(\mathbf{r}-\mathbf{r}^{\prime}\right)$,

$\left\{j^{i}(\mathbf{r}), j^{j}\left(\mathbf{r}^{\prime}\right)\right\}=j^{j}(\mathbf{r}) \partial_{i} \delta\left(\mathbf{r}-\mathbf{r}^{\prime}\right)+j^{i}\left(\mathbf{r}^{\prime}\right) \partial_{j} \delta\left(\mathbf{r}-\mathbf{r}^{\prime}\right)$.

Since $\mathbf{j}=\mathbf{v} \rho$ for an equivalent set of brackets

$\left\{v^{i}(\mathbf{r}), \rho\left(\mathbf{r}^{\prime}\right)\right\}=\partial_{i} \delta\left(\mathbf{r}-\mathbf{r}^{\prime}\right)$, 
$\left\{v^{i}(\mathbf{r}), v^{j}\left(\mathbf{r}^{\prime}\right)\right\}=-\frac{\omega_{i j}(\mathbf{r})}{\rho(\mathbf{r})} \delta\left(\mathbf{r}-\mathbf{r}^{\prime}\right)$,

where

$\omega_{i j}(\mathbf{r})=\partial_{i} v_{j}(\mathbf{r})-\partial_{j} v_{i}(\mathbf{r})$

is called the fluid vorticity.

However, for $\omega_{i j}=0$, which will be considered later, the current algebra (10) and (11) changes slightly to

$\left\{\rho(\mathbf{r}), \rho\left(\mathbf{r}^{\prime}\right)\right\}=0$,

$\left\{j^{i}(\mathbf{r}), \rho\left(\mathbf{r}^{\prime}\right)\right\}=\rho(\mathbf{r}) \partial_{i} \delta\left(\mathbf{r}-\mathbf{r}^{\prime}\right)$,

$\left\{j^{i}(\mathbf{r}), j^{j}\left(\mathbf{r}^{\prime}\right)\right\}=j^{j}(\mathbf{r}) \partial_{i} \delta\left(\mathbf{r}-\mathbf{r}^{\prime}\right)+j^{i}\left(\mathbf{r}^{\prime}\right) \partial_{j} \delta\left(\mathbf{r}-\mathbf{r}^{\prime}\right)$

$$
+\left(\partial_{j} j^{i}(\mathbf{r})-\partial_{i} j^{j}(\mathbf{r})\right) \delta\left(\mathbf{r}-\mathbf{r}^{\prime}\right) \text {. }
$$

Positing the Hamiltonian for a barotropic fluid with potential energy $U(\rho)$ as

$H=\int \mathrm{d} r\left(\frac{1}{2} \rho \mathbf{v}^{2}+U(\rho)\right)$,

the equations of motion turn out to be

$\dot{\rho}=\{H, \rho\}=-\vec{\nabla} \cdot(\mathbf{v} \rho)$,

$\dot{\mathbf{v}}=\{H, \mathbf{v}\}=-(\mathbf{v} \cdot \vec{\nabla}) \mathbf{v}-\vec{\nabla} U^{\prime}(\rho)$.

Equations (19) and (20) constitute, respectively, the continuity equation and Euler (force) equation, the two central equations governing perfect fluid dynamics.

Furthermore the Hamiltonian formalism is very convenient for studying the symmetries of the system. Renaming the densities in a suggestive way for a generalization to relativistic fluid [12], (which will not be pursued here),

$\mathcal{E}=\frac{1}{2} \rho \mathbf{v}^{2}+U=T^{00}$

together with the energy flux

$T^{j o}=\rho v^{j}\left(\frac{1}{2} \mathbf{v}^{2}+U^{\prime}\right)$,

one derives the energy conservation law,

$\dot{T}^{o o}+\partial_{j} T^{j o}=0$.

The momentum density, $\mathcal{P}$,

$\mathcal{P}^{i}=\rho v^{i}=T^{o i}$

and the stress tensor $T^{i j}$ with $P=\rho U^{\prime}-U$ defined as the pressure,

$T^{i j}=\delta^{i j}\left(\rho U^{\prime}-U\right)+\rho v^{i} v^{j}=\delta^{i j} P+\rho v^{i} v^{j}$

satisfy

$\dot{T}^{o i}+\partial_{j} T^{j i}=0$.
The time translation and space translation invariances are ensured by the conserved quantities,

$E=\int \mathrm{d} x \mathcal{E}$ (time-translation),

$\mathbf{P}=\int \mathrm{d} r \overrightarrow{\mathcal{P}}=\int \mathrm{d} r \mathbf{j}$ (space-translation).

Rotational invariance is preserved by the conservation of angular momentum,

$M^{i j}=\int \mathrm{d} r\left(r^{i} \mathcal{P}^{j}-r^{j} \mathcal{P}^{i}\right) \quad$ (spatial rotation).

Furthermore invariance of the non-relativistic theory under Galilean transformation yields the Galileo boost constant of motion,

$\mathbf{B}=t \mathbf{P}-\int \mathrm{d} r \mathbf{r} \rho \quad$ (velocity boost).

The continuity equation provides the total number or mass as the final conserved quantity

$N=\int \mathrm{d} r \rho$ (number).

It is quite natural in fluid dynamics to consider irrotational fluids as a special case so that $\omega_{i j}=0$ and we will also adopt this choice for simplicity but as well as for more serious reasons that will become clear as we proceed. We will compare and contrast these important features of the ideal fluid with the NC generalized fluid model that will be formulated in the next section.

\section{Noncommutative extension of fluid variable brackets}

In NC quantum mechanics it is customary to consider NC space as

$\left[\dot{X}^{i}, X^{j}\right]=(i / m) \delta^{i j}, \quad\left[X^{i}, X^{j}\right]=i \theta^{i j}, \quad\left[\dot{X}^{i}, \dot{X}^{j}\right]=0$.

Note that this is the simplest extension that describes an NC space (for applications see [24-29]). Obviously, as explained in the Introduction, this and similar extensions by nonoperatorial terms, such as $\theta_{i j}$ here, satisfy the Jacobi identity and hence are examples of perfectly consistent bracket structure. Here the antisymmetric NC parameter $\theta_{i j}=-\theta_{j i}$ is constant. From high energy physics perspective, bounds on $\theta\left(\theta \leq(10 \mathrm{TeV})^{-2}\right)$ from experimental limits on modifications to a fermion sector are of $\mathrm{O}(10 \mathrm{TeV}) 2$ available $[52,53]$. Although this is very small keeping in mind present day experimental results its effect might turn out to be significant in a classical macroscopic system such as a fluid. ${ }^{4}$

\footnotetext{
4 Indeed it is possible to consider more general forms of noncommutativity with additional constant terms in rest of the brackets or even consider operatorial forms of NC structures such as Kappa-Minkowski
} 
As advertised in the Introduction and following (8) [12], we introduce the NC algebra among the Lagrangian degrees of freedom as

$$
\begin{aligned}
\left\{\dot{X}^{i}(\mathbf{x}), X^{j}\left(\mathbf{x}^{\prime}\right)\right\} & =\frac{1}{\rho_{0}} \delta^{i j} \delta\left(\mathbf{x}-\mathbf{x}^{\prime}\right), \\
\left\{X^{i}(\mathbf{x}), X^{j}\left(\mathbf{x}^{\prime}\right)\right\} & =\frac{1}{\rho_{0}} \theta^{i j} \delta\left(\mathbf{x}-\mathbf{x}^{\prime}\right), \quad\left\{\dot{X}^{i}(\mathbf{x}), \dot{X}^{j}\left(\mathbf{x}^{\prime}\right)\right\}=0 .
\end{aligned}
$$

Once again this NC algebra satisfies Jacobi identity and is a perfectly consistent example of bracket structure. This is a classical analog of (32) applied to Lagrangian coordinates and a NC generalization of (8). This simple form of NC extension and its subsequent generalization to $\mathrm{NC}$ quantum field theory started after the seminal work of Seiberg and Witten [7] who showed its relevance in certain low energy limits of string theory where the open string end points reside on NC $D$-branes and $\theta^{\mu \nu}$ gets identified with the antisymmetric field $B^{\mu \nu}$. In the same way as was done for the normal fluid [12], the NC algebra (33) generates the NC Euler variable algebra. A form of "exotic plasma" fluid where the particle Lagrangian coordinates are endowed with an "exotic" algebra (that is, effectively a NC system), was studied in [54]. In another approach $[15,16] \mathrm{NC}$ effects, (of the same canonical form used here), were incorporated directly in the form of star products in the fluid action in Kahler parametrization to NC spacetimes. For completeness we show a few steps explicitly. Using the defining equations for the Euler variables from (5, 6), we need to compute

$$
\begin{aligned}
& \left\{\rho(\mathbf{r}), \rho\left(\mathbf{r}^{\prime}\right)\right\} \\
& \quad=\rho_{0}^{2}\left[\int \delta(X(x)-r) \mathrm{d} x, \int \delta\left(X(y)-r^{\prime}\right) \mathrm{d} y\right], \\
& \left\{v_{i}(\mathbf{r}), \rho\left(\mathbf{r}^{\prime}\right)\right\} \\
& \quad=\left[\frac{\int \mathrm{d} x \dot{X}_{i}(x) \delta(X(x)-r)}{\int \mathrm{d} x \delta(X(x)-r)}, \rho_{0} \int \delta\left(X(y)-r^{\prime}\right) \mathrm{d} y\right], \\
& \left\{v_{i}(\mathbf{r}), v_{j}\left(\mathbf{r}^{\prime}\right)\right\} \\
& \quad=\left[\frac{\int \mathrm{d} x \dot{X}_{i}(x) \delta(X(x)-r)}{\int \mathrm{d} x \delta(X(x)-r)}, \frac{\int \mathrm{d} y \dot{X}_{j}(y) \delta\left(X(y)-r^{\prime}\right)}{\int \mathrm{d} y \delta\left(X(y)-r^{\prime}\right)}\right],
\end{aligned}
$$

Footnote 4 continued

algebra or brackets compatible with the Generalized Uncertainty Principle [35-42]. NC fluid dynamics, compatible with a Snyder form [21-23] of operatorial spacetime has been discussed in $[15,16]$. However, for the type of applications we have in mind, the simplest NC extension studied here induces very interesting and nontrivial effects in the fluid model and so other alternatives have not been considered, although these can be studied in our approach. where the NC algebra (33) between the Lagrange particle coordinates has to be used. We obtain

$$
\begin{aligned}
\left\{\rho(\mathbf{r}), \rho\left(\mathbf{r}^{\prime}\right)\right\}= & \theta_{i j} \partial_{i} \rho(\mathbf{r}) \partial_{j} \delta\left(\mathbf{r}-\mathbf{r}^{\prime}\right) \\
\left\{v_{i}(\mathbf{r}), \rho\left(\mathbf{r}^{\prime}\right)\right\}= & \partial_{i} \delta\left(\mathbf{r}-\mathbf{r}^{\prime}\right)-\theta_{j k} \partial_{j} v_{i}(\mathbf{r}) \partial_{k} \delta\left(\mathbf{r}-\mathbf{r}^{\prime}\right) \\
\left\{v_{i}(\mathbf{r}), v_{j}\left(\mathbf{r}^{\prime}\right)\right\}= & \frac{\left(\partial_{j} v_{i}-\partial_{i} v_{j}\right)}{\rho} \delta\left(\mathbf{r}-\mathbf{r}^{\prime}\right) \\
& +\theta_{k l}\left[\partial_{l} \delta\left(\mathbf{r}-\mathbf{r}^{\prime}\right)\left(\frac{\partial_{k}\left(v_{i} v_{j}\right)}{\rho}-v_{i} v_{j} \partial_{k}\left(\frac{1}{\rho}\right)\right)\right. \\
& \left.+\left(\frac{\partial_{k} v_{i} \partial_{l} v_{j}}{\rho}-\partial_{l}\left(v_{i} v_{j}\right) \partial_{k}\left(\frac{1}{\rho}\right)\right) \delta\left(\mathbf{r}-\mathbf{r}^{\prime}\right)\right] \\
& -\frac{\rho_{0} \theta_{k l}}{\rho(\mathbf{r}) \rho\left(\mathbf{r}^{\prime}\right)} \partial_{l} \delta\left(\mathbf{r}-\mathbf{r}^{\prime}\right) \\
& \times \frac{\partial}{\partial r_{k}}\left[\int \mathrm{d} x \dot{X}_{i}(x) \dot{X}_{j}(x) \delta(X(x)-r)\right]
\end{aligned}
$$

Comparing with (12) and (13) we immediately notice that a rich NC algebra has emerged due to the mapping between Lagrange and Euler degrees of freedom, which reduces to the canonical form (12) and (13) for $\theta^{i j}=0$. However, we also encounter a closure problem (which is typical of nonlinear systems) in that the $\left\{v_{i}, v_{j}\right\}$ bracket contains higher moments of $v_{i}$. First of all let us consider an irrotational fluid (in the canonical case, that is, with $\theta_{i j}=0$ ) having zero vorticity $\omega_{i j}=\partial_{i} v_{j}-\partial_{j} v_{i}=0$, by which one gets rid of the canonical part of the $\left\{v_{i}, v_{j}\right\}$ bracket. This simplification is a common practice in various contexts of fluid dynamics. But, interestingly enough, even with this restriction, $O(\theta)$ terms in $\left\{v_{i}(\mathbf{r}), v_{j}\left(\mathbf{r}^{\prime}\right)\right\}$ survive, showing that a noncommutativityinduced vorticity has reappeared. This is reminiscent of our previous result that $\mathrm{NC}$ can induce spin in mechanical systems [55]. Hence, to make life simpler, at least for the present work, we will further assume that $O\left(\theta v^{2} / \rho\right)$ terms are small and omit them, indicating that we are working in a low velocity (or energy) and high density regime. Throughout our work we will exploit the relation $\omega_{i j}=\partial_{i} v_{j}-\partial_{j} v_{i}=0$ many times.

Hence after all these restrictions of irrotational fluid at low velocity and high density we have been able to provide an extended fluid algebra which has been derived from basic principles and has its origin in conventional NC point mechanics. The cherished NC fluid algebra,

$$
\begin{aligned}
& \left\{v_{i}(\mathbf{r}), \rho\left(\mathbf{r}^{\prime}\right)\right\}=\partial_{i} \delta\left(\mathbf{r}-\mathbf{r}^{\prime}\right)-\theta_{j k} \partial_{j} v_{i}(\mathbf{r}) \partial_{k} \delta\left(\mathbf{r}-\mathbf{r}^{\prime}\right), \\
& \left\{\rho(\mathbf{r}), \rho\left(\mathbf{r}^{\prime}\right)\right\}=\theta_{i j} \partial_{i} \rho(\mathbf{r}) \partial_{j} \delta\left(\mathbf{r}-\mathbf{r}^{\prime}\right), \\
& \left\{v_{i}(\mathbf{r}), v_{j}\left(\mathbf{r}^{\prime}\right)\right\}=0,
\end{aligned}
$$

constitute our primary major result. Incidentally, it should be pointed out that a structure similar to (41) appeared earlier in [13] (although the full algebra (40)-(42) was not provided) and furthermore it emerged in a totally different framework in magnetohydrodynamics (as discussed in the Introduction) and was applied in a different context. The rest of the paper 
deals with studying some of the fluid properties compatible with this NC algebra.

Once again it is straightforward to recover the NC current algebra,

$$
\begin{aligned}
\left\{\rho(\mathbf{r}), \rho\left(\mathbf{r}^{\prime}\right)\right\}= & \theta_{k l} \partial_{k} \rho(\mathbf{r}) \partial_{l} \delta\left(\mathbf{r}-\mathbf{r}^{\prime}\right), \\
\left\{j_{i}(\mathbf{r}), \rho\left(\mathbf{r}^{\prime}\right)\right\}= & \rho(\mathbf{r}) \partial_{i} \delta\left(\mathbf{r}-\mathbf{r}^{\prime}\right) \\
& +\left.\theta_{j k}\left[2 j_{i} \frac{\partial_{j} \rho}{\rho}-\partial_{j} j_{i}\right]\right|_{r} \partial_{k} \delta\left(\mathbf{r}-\mathbf{r}^{\prime}\right),
\end{aligned}
$$

$$
\begin{aligned}
& \left\{j_{i}(\mathbf{r}), j_{j}\left(\mathbf{r}^{\prime}\right)\right\} \\
& =\left[\left.\left(j_{i}\left(\delta_{j l}-\theta_{k l} \partial_{k} \frac{j_{j}}{\rho}\right)\right)\right|_{r}+\left.\left(j_{j}\left(\delta_{i l}-\theta_{k l} \partial_{k} \frac{j_{i}}{\rho}\right)\right)\right|_{r^{\prime}}\right] \\
& \quad \times \partial_{l} \delta\left(\mathbf{r}-\mathbf{r}^{\prime}\right) \\
& \quad+\left[\left(\partial_{j} j_{i}-\partial_{i} j_{j}\right)-\theta_{k l} \partial_{l}\left(\frac{1}{\rho}\right)\left(j_{j} \partial_{k} j_{i}-j_{i} \partial_{k} j_{j}\right)\right] \delta\left(\mathbf{r}-\mathbf{r}^{\prime}\right),
\end{aligned}
$$

where the subscripts $r$ and $r^{\prime}$ denote that the arguments of the functions in $\mathrm{t}$ in parentheses are at $r$ and $r^{\prime}$, respectively.

However, before proceeding with a new Poisson-like structure (especially if it is non-linear and operatorial in nature), it is imperative to ensure that it satisfies the Jacobi identities.

Jacobi identity considerations Jacobi identity plays a vital role in the internal consistency of the commutator structure in quantum mechanics or quantum field theory. Although, in terms of commutators, it appears as a trivial identity,

$$
\begin{aligned}
J(A, B, C) & \equiv[[A, B], C]+[[B, C], A]+[[C, A], B] \\
& =0,
\end{aligned}
$$

leading to compatibility with Jacobi identity, the actual situation is much more tricky in quantum field theory due to a clash between singularities and limiting procedures and this requirement yields nontrivial consequences (see for example [56-58]). Its classical analog, defined in terms of Poisson brackets (or Dirac brackets in constrained systems),

$$
\begin{aligned}
J(A, B, C) & \equiv\{\{A, B\}, C\}+\{\{B, C\}, A\}+\{\{C, A\}, B\} \\
& =0
\end{aligned}
$$

imposes an equally important restriction on the brackets. Indeed, for canonical brackets as in (46) as well as its extension by $c$-number functions as in (47) or more generalized versions, the extended NC brackets trivially satisfy $J(A, B, C)=0$ without imposing any restrictions on the extensions. The double commutators will vanish trivially so long as the single commutators do not involve operators on the RHS. However, the situation changes drastically once the brackets involve operators, such as the brackets among the fluid variables given in (40)-(42). Naively one might think that if the canonical algebra (9)-(11) satisfied Jacobi identities (which it does [12]), the present set should also satisfy the Jacobi identities since after all only constant NC extensions have been taken into account in the parent Lagrangian coordinates (33). However, checking that the algebra (40)(42) satisfies Jacobi identities is somewhat nontrivial as we briefly demonstrate below.

For field theory one has the requirement that Jacobi identities have to be obeyed locally:

$$
\begin{aligned}
& J(A(x), B(y), C(z)) \\
& \equiv\{\{A(x), B(y)\}, C(z)\}+\{\{B(y), C(z)\}, A(x)\} \\
& \quad+\{\{C(z), A(x)\}, B(y)\}=0 .
\end{aligned}
$$

Writing in detail for the density variable $\rho(x)$,

$J\{\rho, \rho, \rho\} \equiv\{\{\rho(x), \rho(y)\}, \rho(z)\}+$ cyclic terms,

we find

$$
\begin{aligned}
& \{\{\rho(x), \rho(y)\}, \rho(z)\} \\
& \quad=-\theta^{i j} \theta^{k l} \partial_{k} \rho(z) \partial_{j}^{y} \partial_{i}^{z} \partial_{l}^{z}(\delta(x-y) \delta(y-z))=0,
\end{aligned}
$$

which vanishes due to the support of the RHS only for $\mathbf{x}=$ $\mathbf{y}=\mathbf{z}$ and the antisymmetry of $\theta^{i j}$, thus leading to

$J\{\rho, \rho, \rho\} \equiv\{\{\rho(x), \rho(y)\}, \rho(z)\}+$ cyclic terms $=0$.

In a similar way for the mixed $J\left\{\rho(x), \rho(y), v_{i}(z)\right\}$ we have

$$
\begin{aligned}
& \left\{\{\rho(x), \rho(y)\}, v_{i}(z)\right\} \\
& \quad=-\theta^{k l}\left[\partial_{k}^{y} \partial_{l}^{z} \partial_{i}^{z}(\delta(x-y) \delta(x-z))\right. \\
& \left.\quad+\theta^{m n} \partial_{k} v_{i}(z) \partial_{n}^{y} \partial_{k}^{z} \partial_{l}^{z}(\delta(x-y) \delta(x-z))\right]=0
\end{aligned}
$$

due to support of RHS only for $\mathbf{x}=\mathbf{y}=\mathbf{z}$ and antisymmetry of $\theta^{i j}$. The other two cyclic terms also vanish for the same reason, yielding

$J\left\{\rho, \rho, v_{i}\right\} \equiv\left\{\{\rho(x), \rho(y)\}, v_{i}(z)\right\}+$ cyclic terms $=0$.

The rest of the Jacobi identities $J\left(\rho, v_{j}, v_{i}\right)$ and $J\left(v_{k}, v_{j}, v_{i}\right)$ are trivially satisfied. Thus the new algebra (40)-(42) provided by us proves to be a consistent extension of irrotational fluid dynamics algebra in noncommutative space.

\section{Properties of NC fluid: conservation laws and symmetries}

Let us begin by discussing the symmetries and conservation laws of the NC fluid as a Hamiltonian system.

Conservation laws As a trial form we assume the same expression for the Hamiltonian as the canonical one (18). Exploiting the NC algebra (40)-(42) we compute the NC fluid equations of motion, 


$$
\begin{aligned}
\dot{\rho} & =\{H, \rho\}=-\partial_{i}\left(\rho v_{i}+\theta_{i j} \rho \partial_{j} v^{2}\right) \\
\dot{v}_{i} & =\left\{H, v_{i}\right\}=-\partial_{i}\left(\frac{v^{2}}{2}+U^{\prime}\right)+\theta_{j k}\left(\partial_{i} v_{j}\right) \partial_{k}\left(\frac{v^{2}}{2}+U^{\prime}\right) \\
& =-\partial_{j}\left(\left(\frac{v^{2}}{2}+U^{\prime}\right) \delta^{i j}+\theta_{j k}\left(\frac{v^{2}}{2}+U^{\prime}\right)\left(\partial_{k} v_{i}\right)\right)
\end{aligned}
$$

We stress that in the NC generalized $\rho$-equation of motion (54) can still be interpreted as a conservation law since the $\mathrm{NC}$ correction term also is a total derivative, leading to the conservation of total number $N$ as defined in (31) in the $\mathrm{NC}$ generalization. ${ }^{5}$ In the NC modified Euler equation out of the $\theta$-terms, $\left(\theta_{j k} \partial_{i} v_{j}\right) \partial_{k}\left(\frac{v^{2}}{2}\right)$ can be dropped in the approximation we have considered but $\left(\theta_{j k} \partial_{i} v_{j}\right) \partial_{k} U^{\prime}$ will survive. Later on, in the context of cosmology, we will demonstrate that the $\theta$-terms in these two basic fluid equations provide the seeds for cosmological perturbations.

Next we come to the all-important equation that deals with energy conservation. Exploiting the equations for $\dot{\rho}$ and $\dot{v}_{i}$ from $(54,56)$ and using

$\mathcal{E}=\frac{\rho v^{2}}{2}+U(\rho)$

we obtain

$$
\begin{aligned}
\dot{\mathcal{E}}= & -\partial_{i}\left[\rho v_{i}\left(\frac{v^{2}}{2}+U^{\prime}\right)\right. \\
& \left.+\theta_{i j}\left\{-\partial_{j} v^{2}\left(\rho \frac{v^{2}}{2}+U\right)+\frac{v^{2}}{2} \partial_{j} P\right\}\right],
\end{aligned}
$$

where $P=\rho U^{\prime}-U$ is the pressure. Very interestingly the energy conservation law is maintained since, once again as it happened for the number conservation law in (54), the extra term is a total derivative. Thus the canonical form of energy density is preserved but going back to our earlier identification (22) we find that the energy flux $T^{j 0}$ receives an NC correction,

$$
\begin{aligned}
T^{j 0}= & \rho v_{i}\left(\frac{v^{2}}{2}+U^{\prime}\right) \\
& +\theta_{i j}\left\{-\partial_{j} v^{2}\left(\rho \frac{v^{2}}{2}+U\right)+\frac{v^{2}}{2} \partial_{j}\left(\rho U^{\prime}-U\right)\right\} .
\end{aligned}
$$

Spacetime symmetries Let us now check translation invariance of the NC fluid system. Keeping the expression for momentum $\pi^{i}$ unchanged we define $\Pi^{i}=\int \mathrm{d} r \pi^{i}$ and compute

$\left\{\Pi^{i}, \rho\left(\mathbf{r}^{\prime}\right)\right\}=-\partial_{i} \rho$,

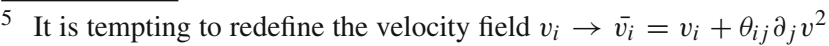
so that (54) can be recast as $\dot{\rho}+\partial_{i}\left(\rho \bar{v}_{i}\right)$ but unfortunately this does not lead to a simpler bracket structure with $\bar{v}_{i}, \rho$ and so we do not consider it.
}

$\left\{\Pi^{i},, v_{j}\left(\mathbf{r}^{\prime}\right)\right\}=-\partial_{i} v_{j}+\theta_{k l} \partial_{k} v_{j} \partial_{l} v_{i}$

Notice that $\Pi$ translates $\rho$ correctly in the NC space but fails to do so for $\mathbf{v}$. However, pursuing with the definition for $\pi_{i}$ we compute its time derivative to find

$$
\begin{aligned}
\pi^{i} & =\rho v_{i}, \\
\dot{\pi}^{i}=\partial_{j} & {\left[-\left\{\rho v_{i} v_{j}+\delta_{i j} P\right\}+\theta_{j k}\left\{v_{i} \partial_{k} P-v_{i} \rho \partial_{k} v^{2}\right\}\right] } \\
& +\frac{3}{2} \theta_{j k} \rho\left(\partial_{j} v_{i}\right) \partial_{k} v^{2} .
\end{aligned}
$$

It is worthwhile to comment on this relation. First of all note that the last term in RHS, not being a total derivative, apparently breaks the total momentum conservation of $\Pi^{i}$ but we can safely drop the disturbing last term in our approximation scheme, leading to a slightly "weaker" momentum conservation principle. But this is to be expected since the $\theta$-correction acts as an external force as we have seen in the modified Euler equation (56).

Thus we end up with

$\dot{\pi}^{i}=\partial_{j}\left(-\left(\rho v_{i} v_{j}+\delta_{i j} P\right)+\theta_{j k} v_{i} \partial_{k} P\right)$,

and we define a modified $T^{i j}$,

$T^{i j}=-\left(\rho v_{i} v_{j}+\delta_{i j} P\right)+\theta_{j k} v_{i} \partial_{k} P$.

We immediately observe that the $\theta$-contribution in $T^{i j}$ is not symmetric under interchange of $i, j$. Let us return to the conservation of angular momentum $M^{i j}$ in the canonical fluid $\left(\theta^{i j}=0\right)$. This requires using the Eqs. $(24,25,26,29)$ together with the fact that (25) is symmetric. Since the NC correction in $T^{i j}$ is not symmetric the total angular momentum will not be conserved for the NC fluid and in particular

$$
\begin{aligned}
\dot{M}_{i j} & =\int \mathrm{d} r\left(r^{i} \dot{\pi}^{j}-r^{j} \dot{\pi}^{i}\right) \\
& =\int \mathrm{d} r \theta_{k l}\left[r^{i} \partial_{j} v_{l}-r^{j} \partial_{i} v_{l}\right]\left(\partial_{k} v^{2}\right) \rho .
\end{aligned}
$$

Again this result is also expected since we have introduced a constant set of parameters $\theta^{i j}$ that do not transform at all under rotations so that the fundamental NC bracket in (33), $\left\{X^{i}(\mathbf{x}), X^{j}\left(\mathbf{x}^{\prime}\right)\right\}=\frac{\theta_{i j}}{\rho_{o}} \delta\left(\mathbf{x}-\mathbf{x}^{\prime}\right)$ fails to transform covariantly under spatial rotation. On the other hand the Galileo boost constant of motion B (30) will be preserved in the NC space in our approximation scheme.

\section{Cosmological implications}

In this section we wish to outline how the $\mathrm{NC}$ extended fluid equations, in particular the NC Euler equation (56), can introduce density perturbations in cosmology (for basic cosmology see for example [59]). There are several NC extensions of cosmological models, such as [60] (where the NC 
geometry is imposed from the spectral point of view [6163]). On the other hand, in [64] noncommutativity is introduced from spacetime uncertainty relation and the formalism requires spacetime noncommutativity in an essential way. (For questions related to the unitarity of models with spacetime noncommutativity see the comment and references in [64].) These models are very different from our way of introducing NC at Lagrangian coordinate level, explained earlier.

Before presenting our formulation of NC gravity-fluid system let us emphasize that we are considering a simplified system where gravity appears in a mean-field approximation. This is because the NC extension of a fully interacting gravity-fluid system is very complicated and we restrict ourselves to a scenario where gravity is external in the sense that it is not affected by the NC modification of the fluid.

However, before proceeding, it is crucial to understand how the $\theta_{\mu \nu}$ form of $\mathrm{NC}$ can (and if at all at least for the present work) affect the pure (Einstein) gravitational sector. In this context, (as mentioned in the Introduction), we need to exploit the other alternative path leading to NC extension, that is, a direct application of the Groenwald-Moyal *product [7,18-20] in Einstein general theory action to obtain the NC-extended field theory action. Now, gravity can be considered as a gauge theory and [7] provides a prescription to generalize a gauge theory in NC space via the SeibergWitten map [7]. But the problem in this naive application is that the fundamental NC structure (32) is not invariant under general coordinate transformation. It was first noticed by Calmet and Kobakidze [65] that there exists a subclass of general coordinate transformations that are compatible with (32) leading to a version of general relativity based on a volumepreserving diffeomorphism. Interestingly, this was already known as the unimodular theory of gravitation [66-69] since the Jacobian of this restricted coordinate transformation is equal to 1 , meaning that the volume is invariant (although spacetime volume may not be preserved).

This model with the gauge symmetries intact was generalized to NC spacetime (via the $*$-product and the SeibergWitten map) but it turned out that due to symmetry requirements the $O(\theta)$ corrections miraculously cancel and hence to first order in $\theta$ the Einstein action and its $\mathrm{NC}$ extension are identical [70-75]. Thus we come to the conclusion that symmetries of canonical noncommutative spacetime naturally lead to the noncommutative version of unimodular gravity for $O(\theta)$ results. Furthermore, there exist other distinct scenarios of NC generalizations, such as discussed in [76] and in all the cases the $O(\theta)$ contribution is absent. Incidentally, the non-zero NC corrections at $O\left(\theta^{2}\right)$ were computed in [77]. In this regard the NC corrections in the matter (fluid) sector presented in our work are linear in $\theta$ and hence we are allowed to work with the cosmological model where the gravity sector remains unchanged and $\mathrm{NC}$ modifications are manifested only in the matter sector.
As is customary in a cosmological context one works in a comoving frame and the starting equations in FriedmannRobertson-Walker (FRW) framework of cosmology are the continuity and Euler equations,

$$
\begin{aligned}
& \dot{\rho}=-3 H(\rho+P)=-3 \frac{\dot{a}}{a}(\rho+P), \\
& \frac{\ddot{a}}{a}=-\frac{\rho+3 P}{6 M^{2}}+\frac{\Lambda}{3} .
\end{aligned}
$$

$P, \Lambda$ denote the pressure and the cosmological constant, respectively, and $a(t)$ is the scale factor. $M=(8 \pi G)^{-1 / 2}$ refers to Newton's constant $G$. Introducing the Hubble parameter $H(t)=\dot{a} / a$ we recover the Friedmann equation,

$\frac{\dot{a}^{2}}{a^{2}}=H^{2}=\frac{\rho}{3 M^{2}}+\frac{\Lambda}{3}-\frac{k}{a^{2}}$.

Let us now connect the above with the fluid dynamics discussed so far. We need to cast the dynamical fluid equations in comoving coordinates defined as

$\mathbf{r}=a(t) \mathbf{x}(t)$,

where $\mathbf{r}(t), \mathbf{x}(t)$, and $a(t)$ denote, respectively, the proper coordinates, comoving coordinates, and the scale factor exhibiting the expansion of the universe. The $\mathbf{r}(t)$ coincides with our (fluid) position coordinate. The general relation between proper and comoving velocities is given by

$\dot{\mathbf{r}}=H(t) \mathbf{r}+a \dot{\mathbf{x}}(t)$.

Hence the "peculiar velocity" $\dot{\mathbf{x}}(t)$ signifies deviation from the perfect Hubble flow. In conventional cosmological FRW model one considers the comoving coordinates $\mathbf{x}(t)$ to be time independent so that the comoving velocity $\dot{\mathbf{x}}(t)$ is zero. In our notation (70) will be

$\mathbf{v}=H(t) \mathbf{r}+\mathbf{u}$,

with $\mathbf{u}$ defined as the peculiar velocity. We rewrite the NC fluid equations once again,

$\left.\frac{\partial \rho}{\partial t}\right|_{r}+\frac{\partial}{\partial r_{i}}\left(\rho v_{i}+\theta_{i j} \rho \partial_{j} v^{2}\right)=0$,

$\left.\frac{\partial v_{i}}{\partial t}\right|_{r}+v_{j} \partial_{j} v_{i}=-\frac{\partial_{i} P}{\rho}+\frac{\theta_{j k} \partial_{i} v_{j} \partial_{k} P}{\rho}-\partial_{i} \Phi$,

where we introduce $\Phi$ as a generic potential. We need to recast the dynamics in the comoving coordinates $\mathbf{x}, t$. The space derivatives are easily related by

$\partial / \partial \mathbf{r}=(1 / a) \partial / \partial \mathbf{x}$.

On the other hand, the time derivatives at constant $\mathbf{r}$ and constant $\mathbf{x}$ are related by

$\left.\frac{\partial}{\partial t}\right|_{\mathbf{r}}=\left.\frac{\partial}{\partial t}\right|_{\mathbf{x}}-\frac{\dot{a}}{a}\left(\mathbf{x} \cdot \partial_{\mathbf{x}}\right)$. 
Furthermore note that the "no vorticity condition" $\omega_{i j}=0$ in comoving coordinates is expressed as

$\frac{1}{a} \frac{\partial}{\partial x_{i}}\left(\dot{a} x_{j}+u_{j}\right)=\frac{1}{a} \frac{\partial}{\partial x_{j}}\left(\dot{a} x_{i}+u_{i}\right)$.

Simplifying the above to

$\dot{a} \delta_{i j}+\frac{\partial u_{j}}{\partial x_{i}}=\dot{a} \delta_{i j}+\frac{\partial u_{i}}{\partial x_{j}}$

we find

$\frac{\partial u_{j}}{\partial x_{i}}=\frac{\partial u_{i}}{\partial x_{j}}$.

This relation will be used subsequently. Exploiting the above identities we derive the cherished expressions for the NC fluid dynamics in comoving frames:

$$
\begin{aligned}
& \left.\frac{\partial \rho}{\partial t}\right|_{x}+\frac{1}{a} \frac{\partial}{\partial x_{i}}\left(\rho u_{i}\right)+\frac{3 \rho \dot{a}}{a}+\frac{2 \theta_{i j}}{a^{2}} \frac{\partial \rho}{\partial x_{i}} \\
& \times\left(\dot{a}^{2} x_{j}+\dot{a} u_{j}+\left(\dot{a} x_{k}+u_{k}\right) \frac{\partial u_{j}}{\partial x_{k}}\right)=0, \\
& \frac{\partial u_{i}}{\partial t}+\frac{\dot{a}}{a} u_{i}+\frac{u_{j}}{a} \frac{\partial u_{i}}{\partial x_{j}}=-\frac{1}{a \rho} \frac{\partial P}{\partial x_{i}}-\frac{1}{a} \frac{\partial \phi_{p e c}}{\partial x_{i}} \\
& +\theta_{i k} \frac{\dot{a}}{\rho a^{2}} \frac{\partial P}{\partial x_{k}}+\theta_{j k} \frac{1}{\rho a^{2}} \frac{\partial u_{j}}{\partial x_{i}} \frac{\partial P}{\partial x_{k}}-\left(\ddot{a}+\frac{4 \pi}{3} a G \rho_{b}\right) x_{i} .
\end{aligned}
$$

Without the peculiar velocity (u-dependent) terms the NC continuity equation (77) reduces to

$\dot{\rho}+\frac{3 \rho \dot{a}}{a}+\psi=0$,

where $\psi=\frac{2 \theta_{i j}}{a^{2}} \frac{\partial \rho}{\partial x_{i}} \dot{a}^{2} x_{j}$ is the NC correction. ${ }^{6}$ On the other hand, the $\mathbf{x}$-dependent term in (78) yields

$\ddot{a}+\frac{4 \pi}{3} a G \rho_{b}=0$,

which agrees with (67) provided $\Lambda=0$ and $P=0$ and a spherically symmetric dust potential is considered, that is,

$\Phi=(2 / 3) \pi G \rho_{b}(a \mathbf{x})^{2}+\phi_{p e c}$.

Here $\phi_{p e c}$ constitutes the peculiar potential. Indeed, for a barotropic fluid whose potential $U(\rho)$ depends on the density $\rho$ only and furthermore specializing to dust, with $U(\rho) \sim \rho$, its pressure vanishes, $P=\rho U^{\prime}-U=0$. (These restrictions can be relaxed in a straightforward way.)

\footnotetext{
6 As an aside we note that the conventional continuity equation for a fluid can be recovered from first law of thermodynamics for adiabatic processes. Hence the NC correction brings in some sort of nonadiabaticity in the process.
}

Let us note the consequences of an NC space. From (79, 80 ) it is clear that to our level of approximation, even the so-called "unperturbed" universe with $\mathbf{u}=0$ receives $\mathrm{NC}$ contributions. From (79) and (80) we recover

$\frac{1}{2} \frac{\mathrm{d}}{\mathrm{d} t}\left(\dot{a}^{2}\right)=\frac{4 \pi G}{3} \frac{\mathrm{d}}{\mathrm{d} t}\left(\rho a^{2}\right)+\frac{4 \pi G}{3} \psi a^{2}$,

leading to a modified Friedmann equation,

$\frac{\dot{a}^{2}}{a^{2}}=\frac{8 \pi G \rho}{3}-\frac{k}{a^{2}}+\frac{8 \pi G}{3} \frac{1}{a^{2}} \int a^{2} \psi \mathrm{d} t$,

where $k$ is a constant. Hence for non-zero pressure we see that the $\mathbf{u}$-equation of motion from the Euler equation (78) will be modified by $\theta$-contributions. We do not wish to dwell too much on NC effects on cosmological (density) perturbations in the present paper as these will be analyzed in a separate publication. But just to indicate a straightforward NC effect, let us express (83) as

$\frac{\dot{a}^{2}}{a^{2}}=\frac{8 \pi G \rho}{3}-\frac{k_{\mathrm{nc}}}{a^{2}}$,

where $k_{\mathrm{nc}}=k-\frac{8 \pi G}{3} \int a^{2} \psi \mathrm{d} t$. Conventionally $k$ (or $k_{\mathrm{nc}}$ ) in the present case dictates the spatial geometry of the universe and the NC effect can affect the spatial geometry in a nontrivial way. One important aspect of these $\mathrm{NC}$ corrections needs to be taken care of since they inherently bring about anisotropy and inhomogeneity. To incorporate these fluctuations in generalized FRW models consistently some spatial averaging hypothesis or prescription (over Lagrangian coordinates) is required. For example in a series of works Buchert [78-80] has developed an averaging prescription as back reaction effects, which might be adopted for our case. Indeed, it is too early in our analysis to comment on the possibility of observing noncommutative effects in the context of cosmology from a numerical feasibility point of view.

\section{Summary and future prospects}

Let us summarize our work. In the first part we have provided a new framework to consider a generalization of a perfect fluid model in noncommutative space. The NC fluid model is developed from first principles. We have introduced the noncommutative algebra in particle coordinates (or equivalently Lagrangian degrees of freedom) for the fluid. Subsequently we have derived the noncommutative algebra for fluid degrees of freedom in Eulerian picture. We have ensured that the new algebra satisfies Jacobi identities. We have shown that the NC fluid model constructed here satisfies total mass (or number $N$ ) and total energy conservation principles. In our scheme the expressions for number density and energy density remain canonical but noncommutative correc- 
tions are manifested in the number current and energy fluxes. The total momentum is conserved in the order of approximation we are interested in. The total angular momentum is also conserved in our level of accuracy, although locally the rotational invariance is not preserved due to the introduction of the constant vectorial NC parameter $\theta^{i j}$.

In the second part we have discussed how the present noncommutative fluid model can influence conventional cosmological principles since the all-important continuity and Euler equations get modified in a nontrivial way. In particular noncommutative corrections introduce inhomogeneity and anisotropy through cosmological perturbations.

Various directions of study open up from the present analysis. Let us list some of them.

Noncommutative fluid theory A natural extension of the present analysis is to consider fluids with non-vanishing vorticity.

Our framework is essentially Hamiltonian but in conventional fluid dynamics there exists an action principle constructed in terms of Clebsch variables. It will be worthwhile to find a Clebsch parameterization for the noncommutative fluid.

The present study is restricted to a non-relativistic setup. Study of Schwinger's condition, a hallmark of relativistic quantum field theory, for non-relativistic fluid models has received attention recently in [49-51]. Similar aspects can be analyzed for the present fluid system as well. Another interesting theme is the entropy consideration. In fact it is possible that already the noncommutative effects have brought about an entropy variable since as we have pointed out it imposes some form of non-adiabaticity in the formalism. It will also be very interesting if the noncommutative fluid can be generalized to a relativistic scenario. Work is in progress along these directions.

Noncommutative fluid in cosmological context In the present work we just indicated how noncommutative space can have influence cosmological context without providing any explicit computations and results. The specific effects of the noncommutativity-induced density perturbations, derived here, can be studied in detail.

Noncommutative fluid and gauge/gravity duality Exploiting the AdS/CFT duality the pioneering work of $[33,34]$ in the context of the gauge/gravity duality discusses the correspondence between relativistic, conformal hydrodynamics and Einstein's theory of gravity. The fundamental role in this context is played by the energy-momentum tensor. The ideal fluid stress tensor does not contain derivatives of velocity and so cannot incorporate dissipative effects. We have pointed out that the noncommutative effects can generate derivative terms in stress tensor components. Thus the extension of fluid dynamics to noncommutative fluid dynamics may lead to new features in the gauge/gravity correspondence.
Acknowledgement The work of P.D. is supported by INSPIRE, DST, India.

Open Access This article is distributed under the terms of the Creative Commons Attribution 4.0 International License (http://creativecomm ons.org/licenses/by/4.0/), which permits unrestricted use, distribution, and reproduction in any medium, provided you give appropriate credit to the original author(s) and the source, provide a link to the Creative Commons license, and indicate if changes were made.

Funded by SCOAP ${ }^{3}$.

\section{References}

1. S. Bahcall, L. Susskind, Int. J. Mod. Phys. B 5, 2735 (1991)

2. L. Susskind. arXiv:hep-th/0101029

3. A. El Rhalami, E.M. Sahraoui, E.H. Saidi, J. High Energy Phys. 05, 004 (2002)

4. J.L.F. Barbon, A. Paredes, Int. J. Mod. Phys. A 17, 3589 (2002)

5. J.L.F. Barbon, D. Gerber, Int. J. Mod. Phys. A 22, 5287 (2007)

6. A.P. Polychronakos, Seminaire Poincare X (Institut Henri Poincare, Paris, 2007) (to be published)

7. N. Seiberg, E. Witten, JHEP 9909, 032 (1999). arXiv:hep-th/9908142

8. R. Jackiw, Physical instances of noncommuting coordinates. Nucl. Phys. Proc. Suppl. 108, 30-36 (2002)

9. R. Jackiw, Phys. Part. Nucl. 33, S6-S11 (2002)

10. R. Jackiw, Lect. Notes Phys. 616, 294-304 (2003). arXiv:hep-th/0110057

11. R. Jackiw, S.Y. Pi, A.P. Polychronakos, Ann. Phys. 301, 157-173 (2002). arXiv:hep-th/0206014

12. R. Jackiw, V.P. Nair, S.-Y. Pi, A.P. Polychronakos, J. Phys. A 37, R327-R432 (2004). arXiv:hep-ph/0407101

13. Z. Guralnik, R. Jackiw, S.-Y. Pi, A. Polychronakos, Phys. Lett. B $\mathbf{5 1 7}, 450(2001)$

14. L. O'Raifeartaigh, fluids, and noncommuting fields. arXiv:physics/0209108

15. M.C.B. Abdalla, L. Holender, M.A. Santos, I.V. Vancea, Phys. Rev. D 86, 045019 (2012)

16. L. Holender, M.A. Santos, M.T.D. Orlando, I.V. Vancea, Phys. Rev. D 84, 105024 (2011)

17. M.V. Marcial, A.C.R. Mendes, C. Neves, W. Oliveira, F.I. Takakura, Phys. Lett. A 374, 3608 (2010)

18. M.R. Douglas, N.A. Nekrasov, Rev. Mod. Phys. 73, 977 (2001). arXiv:hep-th/0106048

19. R.J. Szabo, Phys. Rep. 378, 207 (2003). arXiv:hep-th/0109162

20. R. Banerjee, B. Chakraborty, S. Ghosh, P. Mukherjee, S. Samanta, Found. Phys. 39, 1297-1345 (2009). arXiv:0909.1000

21. H.S. Snyder, Phys. Rev. 71, 38 (1947)

22. H.S. Snyder, Phys. Rev. 72, 68 (1947)

23. C.N. Yang, Phys. Rev. 72, 874 (1947)

24. J. Gamboa, M. Loewe, J.C. Rojas, Phys. Rev. D 64, 067901 (2001)

25. V.P. Nair, A.P. Polychronakos, Phys. Lett. B 505, 267-274 (2001). arXiv:hep-th/0011172

26. H.O. Girotti, Am. J. Phys. 72, 608 (2004)

27. S. Ghosh, P. Roy. doi:10.1016/j.physletb.2012.04.033. arXiv: 1110.5136 [hep-th]

28. S.A. Alavi, S. Abbaspour, J. Phys. A 47, 045303 (2014). doi:10. 1088/1751-8113/47/4/045303. arXiv:1304.6160 [hep-th]

29. O. Bertolami, P. Leal, Phys. Rev. D 750, 6 (2015). arXiv:hep-th/0010220

30. J. Maldacena et al., Phys. Rept. 323, 183 (2000)

31. S.S. Gubser, Phys. Rev. D 78, 065034 (2008)

32. S.A. Hartnoll, C.P. Herzog, G.T. Horowitz, Phys. Rev. Lett. 101, 031601 (2008) 
33. S. Bhattacharyya, V.E. Hubeny, S. Minwalla, M. Rangamani, JHEP 02, 045 (2008), arXiv:0712.2456

34. S. Bhattacharyya, V.E. Hubeny, R. Loganayagam, G. Mandal, S. Minwalla, T. Morita, M. Rangamani, H.S. Reall, JHEP 2008(6), 055 (2008). arXiv:0803.2526v2 [See for example N. Ambrosetti, J. Charbonneau, S. Weinfurtner, The fluid, gravity correspondence: Lectures notes from the, Summer School on Particles, Fields, and Strings (UBC, Canada, 2008)]

35. A. Kempf, J. Phys. A 30, 2093 (1997). arXiv:hep-th/9604045

36. A. Kempf, G. Mangano, R.B. Mann, Phys. Rev. D 52, 1108 (1995). arXiv:hep-th/9412167

37. A. Kempf, G. Mangano, Phys. Rev. D 55, 7909 (1997). arXiv:hep-th/9612084

38. G. Amelino-Camelia, Nature 418, 34 (2002)

39. G. Amelino-Camelia, Phys. Lett. B 510, 255 (2001)

40. G. Amelino-Camelia, Int. J. Mod. Phys. A 11, 35 (2002)

41. J. Kowalski-Glikman, S. Nowak, Phys. Lett. B 539, 126 (2002). arXiv:hep-th/0203040

42. S. Das, E.C. Vagenas, Phys. Rev. Lett. 101, 221301 (2008). arXiv:0810.5333

43. S. Pramanik, S. Ghosh, Int. J. Mod. Phys. A 28, 1350131 (2013). arXiv: 1301.4042

44. S. Pramanik, S. Ghosh, P. Pal, Ann. Phys. 346, 113 (2014). arXiv: 1212.6881

45. S. Ghosh, P. Pal, Phys. Rev. D 75, 105021 (2007). arXiv:hep-th/0702159

46. S. Ghosh, Phys. Rev. D 74, 084019 (2006). arXiv:hep-th/0608206

47. A.A. Deriglazov, W.G. Ramrez, Phys. Rev. D 92, 124017 (2015). arXiv: 1509.04926

48. P.A.M. Dirac, Lectures on Quantum Mechanics (Yeshiva University Press, New York, 1964)

49. R. Banerjee, S. Ghosh, A.K. Mitra, EPJC 75, 207 (2015). arXiv: 1409.7175

50. R. Banerjee, A.K. Mitra. arXiv:1510.00288

51. R. Banerjee, S. Ghosh, A.K. Mitra. arXiv:1604.06544

52. M. Chaichian, M. Sheikh-Jabbari, A. Tureanu, Phys. Rev. Lett. 86, $2716(2001)$

53. S. Carroll, J. Harvey, V.A. Kostelecky, C. Lane, T. Okamoto. arXiv:hep-th/0105082

54. C. Duval, Z. Horváth, P.A. Horváthy, Int. J. Mod. Phys. B 15, 3397 (2001). arXiv:cond-mat/010144
55. S. Ghosh, Mod. Phys. Lett. A 20, 1227-1238 (2005). doi:10.1142/ S0217732305017494. arXiv:hep-th/0407086

56. S. Ghosh, R. Banerjee, Phys. Lett. B 220, 581 (1989)

57. S. Ghosh, R. Banerjee, Mod. Phys. Lett. A 4, 855 (1989)

58. S. Ghosh, R. Banerjee, Z. Phys. C 41, 121 (1988)

59. A.R. Liddle, D.H. Lyth, Cosmological Inflation and Large-Scale Structure (Cambridge University Press, Cambridge, 2000)

60. M. Marcolli, Noncommutative Geometry and Cosmology. arXiv:0908.3683

61. A. Connes, Commun. Math. Phys. 182(1), 155-176 (1996)

62. A. Connes, Noncommutative Geometry (Academic Press, USA, 1994)

63. A. Chamseddine, A. Connes, M. Marcolli, Adv. Theor. Math. Phys. 11(6), 991-1089 (2007)

64. R. Brandenberger, P.M. Ho, Phys. Rev. D 66, 023517 (2002). arXiv:hep-th/0203119

65. X. Calmet, A. Kobakhidze, Phys. Rev. D 72, 045010 (2005). arXiv:hep-th/0506157

66. J.J. van der Bij, H. van Dam, Y.J. Ng, Physica 116A, 307 (1982) [further developed by a number of authors. See e.g., F. Wilczek. Phys. Rep. 104, 143 (1984)]

67. W. Buchmuller, N. Dragon, Phys. Lett. B 207, 292 (1988)

68. M. Henneaux, C. Teitelboim, Phys. Lett. B 222, 195 (1989)

69. W.G. Unruh, Phys. Rev. D 40, 1048 (1989)

70. A.H. Chamseddine, Phys. Lett. B 504, 33 (2001)

71. M.A. Cardella, D. Zanon, Class. Quantum Gravity 20, L95 (2003)

72. P. Mukherjee, A. Saha, Phys. Rev. D 74, 027702 (2006)

73. M. Chaichian, A. Tureanu, G. Zet, Phys. Lett. B. 660, 573 (2008)

74. M. Kober, Class. Quantum Gravity 28, 225021 (2011)

75. J. Lukierski, Int. J. Mod. Phys. D 20, 1961 (2011)

76. L. Alvarez-Gaume, F. Meyer, M.A. Vazquez-Mozo, Nucl. Phys. B 753, 92-127 (2006). arXiv:hep-th/0605113

77. X. Calmet, A. Kobakhidze, Phys. Rev. D 74, 047702 (2006). arXiv:hep-th/0605275

78. T. Buchert, ASP Conference Series, 349-356 (1996). arXiv:astro-ph/9512107

79. T. Buchert, Gen. Rel. Gravity 32, 105-125 (2000). arXiv:gr-qc/9906015

80. T. Buchert, Gen. Rel. Gravity 33, 1381-1405 (2001). arXiv:gr-qc/0102049 